\title{
Singular Eigenfunctions of Calogero-Sutherland Type Systems and How to Transform Them into Regular Ones ${ }^{\star}$
}

\author{
Edwin LANGMANN
}

Theoretical Physics, KTH Physics, AlbaNova, SE-106 91 Stockholm, Sweden

E-mail: langmann@kth.se

URL: http://www. theophys.kth.se/ langmann/

Received November 02, 2006, in final form January 29, 2007; Published online February 26, 2007

Original article is available at http://www.emis.de/journals/SIGMA/2007/031/

\begin{abstract}
There exists a large class of quantum many-body systems of Calogero-Sutherland type where all particles can have different masses and coupling constants and which nevertheless are such that one can construct a complete (in a certain sense) set of exact eigenfunctions and corresponding eigenvalues, explicitly. Of course there is a catch to this result: if one insists on these eigenfunctions to be square integrable then the corresponding Hamiltonian is necessarily non-hermitean (and thus provides an example of an exactly solvable $\mathcal{P} \mathcal{T}$-symmetric quantum-many body system), and if one insists on the Hamiltonian to be hermitean then the eigenfunctions are singular and thus not acceptable as quantum mechanical eigenfunctions. The standard Calogero-Sutherland Hamiltonian is special due to the existence of an integral operator which allows to transform these singular eigenfunctions into regular ones.
\end{abstract}

Key words: quantum integrable systems; orthogonal polynomials; singular eigenfunctions

2000 Mathematics Subject Classification: 81U15; 33C50; 05E05

\section{Prologue}

In summer 2003 Stefan Rauch informed me that a colleague of us would soon visit him in Linköping and thus pass through Stockholm, and he suggested that I should invite him for a seminar: this colleague was also working on quantum integrable systems of Calogero-Sutherland (CS) type [1, 2] and I would want to talk to him. I thus got to know Vadim Kuznetsov on September 1 in 2003, short before his seminar where he explained to us their work on the $Q$ operator and separation of variables for the CS model [3]. Stefan Rauch was right: not only did I find the seminar very inspiring and interesting, but also in the discussion afterward I learned about a (for me) new motivation for studying CS type systems which, since then, has influenced and helped me significantly in my work on this topic. By 'learned' I mean here that some way of looking at things, known only from hear-say before and very different from my own, suddenly 'sank in' and made me see things from a new angle. This was a rare experience for me which, I felt, was due to a special channel of communication and mutual understanding I had when talking to Vadim. Vadim was enthusiastic about our science (not only then but in all the, unfortunately too few, instances I had the privilege to meet him), his enthusiasm spread to me, and this was a main reason why I soon afterward returned to my work on the CS system [4, 5] and thus stumbled over results much more extensive and beautiful than what I had expected and for which I am very grateful (these results were announced in $[6,7]$, and the final version of

${ }^{\star}$ This paper is a contribution to the Vadim Kuznetsov Memorial Issue 'Integrable Systems and Related Topics'. The full collection is available at http://www.emis.de/journals/SIGMA/kuznetsov.html 
the detailed paper on this, by which I intend to replace [8], is still in the stage of being 'nearly finished' since 20 months. I miss Vadim's interest and kind encouragement, but I intend to finish it soon, I want to make it as good as I can, and when eventually finished it will be to a large degree Vadim's paper since it would not exist without him).

In the present paper I will present a story which I had carried with me since quite a while (my old draft on it which I use when writing this paper was dated in October 2000) without being able to fully make sense of it. It is about a seemingly provocative result, closely related to this above-mentioned method to construct explicit formulas for eigenfunctions of such systems [7], and I discussed it with Vadim on March 2 (or 3) in 2005, shortly after a seminar to which Vadim had invited me to explain them my approach. I brought up this story in response to a question of Vadim, and after our discussion I understood its place and it finally made sense to me. I remember Vadim liked this story, and this is why I choose it for the present article. Moreover, as will become clear later, this story is related in spirit to Bäcklund transformations and the $Q$-operator which have played a central role in Vadim's research; see e.g. $[9,10]$.

My plan for the rest of this paper is as follows. In the next section I will summarize some known facts about CS systems which are relevant to my story. In Section 3.1 I will present the 'strange' exact eigenfunctions for a large class of generalized CS-type systems, and in Sections 3.2 and 3.3 I clarify their nature by pointing out a natural interpretation in the context of $\mathcal{P} \mathcal{T}$-symmetric mechanics [11]: they provide non-hermitean $\mathcal{P} \mathcal{T}$-invariant quantum many-body Hamiltonians with real spectrum and computable eigenvalues and eigenfunctions. In Section 4 I will explain that these strange eigenfunctions for the CS differential operator are interesting and useful since they can be transformed into regular eigenfunctions. This provides an alternative approach to results previously obtained in [7]. I will end with a few remarks in Section 5. Technical details and some more technical proofs are deferred to three appendices.

\section{Background}

\subsection{CS model and Jack polynomials}

The Calogero-Sutherland (CS) model $[1,2]$ is defined by the differential operator

$$
H_{N}=-\sum_{j=1}^{N} \frac{\partial^{2}}{\partial x_{j}^{2}}+2 \lambda(\lambda-1) \sum_{j<k} \frac{1}{4 \sin ^{2}\left(\frac{1}{2}\left(x_{j}-x_{j}\right)\right)}
$$

with $x_{j} \in[-\pi, \pi]$ coordinates on a circle, $N=2,3, \ldots$, and $\lambda>0$. This differential operator essentially defines a quantum mechanical model of $N$ identical particles moving on a circle and interacting with the $1 / \sin ^{2}$ two-body potential, and the parameter $\lambda$ determines the coupling strength. The CS model is famous among theoretical physicists since it can be solved exactly: the differential operator $H_{N}$ has exact eigenfunctions of the form

$$
\Psi_{\mathbf{n}}(\mathbf{x})=\Psi_{0}(\mathbf{x}) P_{\mathbf{n}}(\mathbf{z})
$$

which are labeled by partitions $\mathbf{n}$ of length $N$, i.e., $\mathbf{n}=\left(n_{1}, n_{2}, \ldots, n_{N}\right)$ with $n_{j}$ integers such that

$$
n_{1} \geq n_{2} \geq \cdots \geq n_{N} \geq 0
$$

and where $P_{\mathbf{n}}$ are certain symmetric polynomials of degree $|\mathbf{n}|=\sum_{j=1}^{N} n_{j}$ in the variables

$$
z_{j}=\mathrm{e}^{\mathrm{i} x_{j}}
$$


with

$$
\Psi_{0}(\mathbf{x})=\prod_{j<k} \sin \left(\frac{1}{2}\left(x_{j}-x_{k}\right)\right)^{\lambda}
$$

the groundstate eigenfunction. Moreover, the corresponding exact eigenvalues have the following remarkably simple form,

$$
E_{\mathbf{n}}=\sum_{j=1}^{N}\left(n_{j}+\frac{1}{2}(N+1-2 j) \lambda\right)^{2} .
$$

The functions $P_{\mathbf{n}}(\mathbf{z})$ are called Jack polynomials and can be characterized, somewhat informally, as follows

$$
P_{\mathbf{n}}(\mathbf{z})=\left(\sum_{P} z_{1}^{P n_{1}} z_{2}^{P n_{2}} \cdots z_{N}^{P n_{N}}\right)+\text { lower order terms }
$$

where the sum is over all distinct permutations $P$ of $\left(n_{1}, n_{2}, \ldots, n_{N}\right)$; see e.g. [12] for a precise characterization.

The following two remarks are on technicalities which can be often safely ignored, but I regard them useful as preparation for our discussion of the generalized CS models in the next section.

Remark 1. For non-integer $\lambda$ our definition of $\Psi_{0}(\mathbf{x})$ above is only complete in the wedge $-\pi<x_{1}<x_{2}<\cdots<x_{N}<\pi$ where we require it to be real (since the phase factors $(-1)^{\lambda}$ obtained by permuting the arguments are obviously ambiguous). There is one natural method to fix this ambiguity by analytical continuation: extend the definition of $\Psi_{0}(\mathbf{x})$ to the other regions by continuing the $x_{j}$ to the complex plane, $x_{j} \rightarrow x_{j}+\mathrm{i} \epsilon j$ with $\epsilon>0$, performing the necessary permutation, and then taking the limit $\epsilon \rightarrow 0$.

Remark 2. It is worth mentioning another seemingly technical point which, however, will play an important role for us later: our 'definition' of the CS model above by $H_{N}$ in (1) was somewhat vague since, to be precise, this quantum mechanical model is defined by a particular self-adjoint operator on the Hilbert space $L^{2}\left([-\pi, \pi]^{N}\right)$. Such an operator is only uniquely defined if one specifies an operator domain, and the latter we did implicitly by characterizing the eigenfunctions. To see that this is not only a technicality we note that the CS model is specified by the parameter $\lambda$, but the coupling constant

$$
\gamma=2 \lambda(\lambda-1)
$$

in (1) is invariant under $\lambda \rightarrow 1-\lambda$. Thus in the coupling regime $-1 / 2<\gamma<0$ one and the same CS differential operator corresponds to two different CS models. In fact, the differential operator in (1) has many more self-adjoint extensions. We will later encounter other examples where one and the same differential operator defines different Hilbert space operators.

We note that these Jack polynomials are just one example in a whole zoo of symmetrical polynomials related to eigenfunctions of CS-type models and which naturally generalize the classical orthogonal polynomials (like Hermite, Laguerre, Jacobi, ... ) to the many-variable case. These polynomials were, and still are, extensively studied in mathematics, and many beautiful results have been discovered; see e.g. [13] and references therein. It was only due to discussions with Vadim that I became aware that my results could be relevant in that context, and it was he who convinced me to write [7] where I could obtain explicit formulas for the Jack polynomials which (to my knowledge) were not know before. One aim of the present paper is to give an alternative derivation and interpretation of these formulas. To be specific I restrict my discussion to the Jack polynomials and only mention in passing that all results allow for a (rather) straightforward generalization to the other symmetric polynomials mentioned above $[14,15]$. 


\section{$2.2 \quad$ A generalized CS model}

There exists a generalization of the CS model describing distinguishable particles and which is partially solvable in the sense that is has a groundstate eigenfunctions and corresponding groundstate energy which can be computed explicitly. This model is defined by the differential operator

$$
\mathcal{H}_{N}=-\sum_{j=1}^{N} \frac{1}{M_{j}} \frac{\partial^{2}}{\partial x_{j}^{2}}+\sum_{j<k} \frac{\gamma_{j k}}{4 \sin ^{2}\left(\frac{1}{2}\left(x_{j}-x_{k}\right)\right)}
$$

with arbitrary mass parameters $M_{j} / 2>0$ and the coupling constants

$$
\gamma_{j k}=\left(M_{j}+M_{k}\right) \lambda\left(M_{j} M_{k} \lambda-1\right),
$$

and it has the following exact groundstate

$$
\Phi_{0}(\mathbf{x})=\prod_{j<k} \sin \left(\frac{1}{2}\left(x_{k}-x_{j}\right)\right)^{\lambda M_{j} M_{k}}
$$

with corresponding groundstate energy

$$
\mathcal{E}_{0}=\frac{\lambda^{2}}{12}\left(\left(\sum_{j=1}^{N} M_{j}\right)^{3}-\sum_{j=1}^{N} M_{j}^{3}\right),
$$

i.e., $\mathcal{H}_{N} \geq \mathcal{E}_{0}$ and

$$
\mathcal{H}_{N} \Phi_{0}(\mathbf{x})=\mathcal{E}_{0} \Phi_{0}(\mathbf{x})
$$

This fact is known since quite a while (see e.g. [16]), but due to its importance for us a short proof is given in Appendix A. This fact plays a twofold role in our story: firstly, it suggests that the Hamiltonian in (6) might have further exactly computable eigenstates, and the search for these led to the result presented in the next section, and secondly, it implies, as special case, the following result which will play an important role for us in Section 4:

Lemma 1. The function

$$
F_{N}(\mathbf{x} ; \mathbf{y})=c \mathrm{e}^{\mathrm{i} P \sum_{j=1}^{N}\left(x_{j}-y_{j}\right)} \frac{\prod_{1 \leq j<k \leq N} \sin \left((1 / 2)\left(x_{j}-x_{k}\right)\right)^{\lambda}}{\prod_{1 \leq j<k \leq N} \sin \left((1 / 2)\left(y_{j}-y_{k}\right)\right)^{\lambda}}
$$

with $x_{j}, y_{j}$ complex variables, $c$ and $P$ arbitrary constants, and the CS Hamiltonian $H_{N}=H_{N}(\mathbf{x})$ in (1), obey the following identity,

$$
H_{N}(\mathbf{x}) F_{N}(\mathbf{x}, \mathbf{y})=H_{N}(\mathbf{y}) F_{N}(\mathbf{x}, \mathbf{y}) .
$$

(The proof is given in Appendix B.1.)

As was pointed out to me by Vadim, the identity in (11) is also implied by a well-known generating function of the Jack polynomials which is closely related to the functions $F_{N}$; see Proposition 2.1 in [12]. We will use this identity to construct an integral transform $\hat{F}_{N}$ in Section 4 which commutes with the CS Hamiltonian and which, for this very reason, will be useful for us. This integral transform is similar to the $Q$-operator defined and exploited in [3], and the usefulness of defining this integral transform $\hat{F}_{N}$ was suggested to me by Vadim. 


\section{Strange exact eigenfunctions of CS-type system}

In this section we construct and discuss singular eigenfunctions of the CS-type differential operator in (6). We note that this result highlights the importance of square integrability in quantum mechanics: if one ignores this condition it is actually easy to construct explicit eigenfunctions for a large variety of models. We first will discuss the simplest class of eigenfunction (Section 3.1). Section 3.2 contains an interpretation of these as eigenfunctions of a non-hermitean Hilbert space operator with purely real spectrum. We also sketch a generalization of this construction illustrating that one and the same differential operator can define a large class of different Hilbert space operators.

\subsection{Construction of singular eigenfunction}

We now construct exact eigenfunctions of the differential operator in (6) with arbitrary coupling constants $\gamma_{j k}$. These eigenfunctions are labeled by integer vectors $\mathbf{n} \in \mathbb{Z}^{N}$ and are linear combinations of the monomials

$$
\check{f}_{\mathbf{n}}(\mathbf{z}):=z_{1}^{n_{1}^{+}} z_{2}^{n_{2}^{+}} \cdots z_{N}^{n_{N}^{+}}, \quad n_{j}^{+}=n_{j}+s_{j}
$$

where $\mathbf{s} \in \mathbb{R}^{N}$ is arbitrary for now. It is important to note that these eigenfunctions are not symmetric, i.e., not invariant under permutations of the particles. Moreover, we will find that these eigenfunctions are only well-defined in a region contained in the following domain,

$$
\Omega_{N}=\left\{\mathbf{z} \in \mathbb{C}^{N}|| z_{1}|<| z_{2}|<\cdots<| z_{N} \mid\right\}
$$

and thus are, in particular, not elements in the Hilbert space $L^{2}\left([-\pi, \pi]^{N}\right) .{ }^{1}$ To state our result we will also need the following partial ordering of integer vectors

$$
\mathbf{m} \preceq \mathbf{n} \Leftrightarrow m_{j}+m_{j+1}+\cdots+m_{N} \leq n_{j}+n_{j+1}+\cdots+n_{N} \quad \forall j=1,2, \ldots, N,
$$

and we will use the special integer vectors

$$
\mathbf{E}_{j k}=\mathbf{e}_{j}-\mathbf{e}_{k}
$$

with $\mathbf{e}_{j}$ the standard basis vectors in $\mathbb{Z}^{N}$, i.e., $\left(\mathbf{e}_{j}\right)_{\ell}=\delta_{j \ell}$ for all $j, \ell=1,2, \ldots, N$. We will also use the notation $\delta_{\mathbf{n}}(\mathbf{m})$ for the Kronecker delta in $\mathbb{Z}^{N}$, i.e.,

$$
\delta_{\mathbf{n}}(\mathbf{m})=\delta_{n_{1}, m_{1}} \delta_{n_{2}, m_{2}} \cdots \delta_{n_{N}, m_{N}} .
$$

Moreover, we will use the following subset of $\mathbb{Z}^{N}$ :

$$
C_{-}^{N}=\left\{\hat{\boldsymbol{\mu}}=\sum_{j<k} \mu_{j k} \mathbf{E}_{j k} \mid \mu_{j k}=0,1,2, \ldots\right\} .
$$

Note that $\mathbf{n}+\hat{\boldsymbol{\mu}} \preceq \mathbf{n}$ for all $\hat{\boldsymbol{\mu}} \in C_{-}^{N}$ and $\mathbf{n} \in \mathbb{Z}^{N}$.

We start our construction by the following simple observation:

Lemma 2. In the region $\Omega_{N}$ the differential operator in (6), for arbitrary $\gamma_{j k}$, acts on the functions in (12) as follows,

$$
\mathcal{H}_{N} \check{f}_{\mathbf{n}}=E_{\mathbf{n}} \check{f}_{\mathbf{n}}-\sum_{j<k} \gamma_{j k} \sum_{\nu=1}^{\infty} \nu \check{f}_{\mathbf{n}+\nu \mathbf{E}_{j k}}
$$

where

$$
E_{\mathbf{n}}=\sum_{j=1}^{N} \frac{\left(n_{j}+s_{j}\right)^{2}}{M_{j}} .
$$

\footnotetext{
${ }^{1}$ In Section 3.2 we will discuss an alternative interpretation of these functions as elements in this Hilbert space.
} 
Proof. Using

$$
-\mathrm{i} \frac{\partial}{\partial x_{j}}=z_{j} \frac{\partial}{\partial z_{j}} \quad \text { and } \quad \frac{1}{4 \sin ^{2}\left(1 / 2\left(x_{j}-x_{k}\right)\right)}=-\frac{z_{j} / z_{k}}{\left(1-z_{j} / z_{k}\right)^{2}}=-\sum_{\nu=1}^{\infty} \nu\left(\frac{z_{j}}{z_{k}}\right)^{\nu}
$$

for $\left|z_{j} / z_{k}\right|<1$, we can write the differential operator in (6) on the domain $\Omega_{N}$ as follows,

$$
\mathcal{H}_{N}=\sum_{j=1}^{N} \frac{1}{M_{j}}\left(z_{j} \frac{\partial}{\partial z_{j}}\right)^{2}-\sum_{j<k} \gamma_{j k} \sum_{\nu=1}^{\infty} \nu\left(\frac{z_{j}}{z_{k}}\right)^{\nu} .
$$

This implies the result.

Equation (13) shows that the action of $\mathcal{H}_{N}$ on the functions $\check{f}_{\mathbf{n}}$ has triangular structure in the following sense: $\mathcal{H}_{N} \check{f}_{\mathbf{n}}$ is a linear superposition of functions $\check{f}_{\mathbf{m}}$ with $\mathbf{m} \preceq \mathbf{n}$. This suggests that $\mathcal{H}_{N}$ should have eigenfunctions $\check{P}_{\mathbf{n}}$ which can be expanded as linear combinations of functions $\check{f}_{\mathbf{m}}, \mathbf{m} \preceq \mathbf{n}$, and with eigenvalues $E_{\mathbf{n}}$. This turns out to be the case, and one finds that the corresponding expansion coefficients can be computed by diagonalizing a triangular matrix if a certain non-degeneracy condition is fulfilled, and that this matrix is simple enough to allow for a fully explicit construction of these eigenfunctions. It is also possible to establish a necessary condition for absolute convergence of this series. To summarize:

Proposition 1. Let $E_{\mathbf{n}}$ be as in (14),

$$
b_{\mathbf{n}}(\mathbf{m})=E_{\mathbf{m}}-E_{\mathbf{n}}=\sum_{j=1}^{N} \frac{1}{M_{j}}\left(m_{j}-n_{j}\right)\left(m_{j}+n_{j}+2 s_{j}\right),
$$

and $\mathbf{n} \in \mathbb{Z}^{N}, \mathbf{s} \in \mathbb{R}^{N}$ and $M_{j}>0$ such that

$$
\left|b_{\mathbf{n}}(\mathbf{n}+\hat{\boldsymbol{\mu}})\right|>\Delta \quad \forall \hat{\boldsymbol{\mu}} \in C_{-}^{N}
$$

for some $\Delta>0$. Then the function

$$
\check{P}_{\mathbf{n}}=\check{f}_{\mathbf{n}}+\sum_{\mathbf{m} \prec \mathbf{n}} \alpha_{\mathbf{n}}(\mathbf{m}) \check{f}_{\mathbf{m}}
$$

with

$$
\alpha_{\mathbf{n}}(\mathbf{m})=\delta_{\mathbf{n}}(\mathbf{m})+\sum_{s=1}^{\infty} \prod_{r=1}^{s}\left(\sum_{j_{r}<k_{r}} \gamma_{j_{r} k_{r}} \sum_{\nu_{r}=1}^{\infty} \nu_{r}\right) \frac{\delta_{\mathbf{n}}\left(\mathbf{m}-\sum_{r=1}^{s} \nu_{r} \mathbf{E}_{j_{r} k_{r}}\right)}{\prod_{r=1}^{s} b_{\mathbf{n}}\left(\mathbf{n}+\sum_{\ell=1}^{r} \nu_{\ell} \mathbf{E}_{j_{\ell} k_{\ell}}\right)}
$$

is an exact eigenfunction of the differential operator in (6) corresponding to the eigenvalue $E_{\mathbf{n}}$ in (14). Moreover, the infinite series defining this function is absolutely convergent in the region where

$$
\sum_{j<k}\left|\gamma_{j k}\right| \frac{\left|z_{j} / z_{k}\right|}{\left(1-\left|z_{j} / z_{k}\right|\right)^{2}}<\Delta
$$

Proof. Inserting the ansatz (17) in the eigenvalue equation $\left(\mathcal{H}_{N}-E\right) \check{P}_{\mathbf{n}}=0$, using (13), and renaming one summation variable we obtain

$$
\sum_{\mathbf{m} \preceq \mathbf{n}}\left(\left(E_{\mathbf{m}}-E\right) \alpha_{\mathbf{n}}(\mathbf{m})-\sum_{j<k} \gamma_{j k} \sum_{\nu=1}^{\infty} \nu \alpha_{\mathbf{n}}\left(\mathbf{m}-\nu \mathbf{E}_{j k}\right)\right) \check{f}_{\mathbf{m}}=0
$$


with $\alpha_{\mathbf{n}}(\mathbf{n})=1$. Since the functions $\check{f}_{\mathbf{m}}$ are linearly independent we conclude that this is true if and only if

$$
\left(E_{\mathbf{m}}-E\right) \alpha_{\mathbf{n}}(\mathbf{m})-\sum_{j<k} \gamma_{j k} \sum_{\nu=1}^{\infty} \nu \alpha_{\mathbf{n}}\left(\mathbf{m}-\nu \mathbf{E}_{j k}\right)=0
$$

for all $\mathbf{m} \preceq \mathbf{n}$. Since $\alpha_{\mathbf{n}}(\mathbf{m})=0$ for $\mathbf{m}>\mathbf{n}$, solving the latter equation amounts to computing a particular eigenvector of a triangular matrix indexed by integer vectors, as anticipated (see Appendix C). We conclude $E=E_{\mathbf{n}}$ and that the coefficients $\alpha_{\mathbf{n}}(\mathbf{m})$ can be computed from the following recursion relations

$$
\alpha_{\mathbf{n}}(\mathbf{m})=\frac{1}{b_{\mathbf{n}}(\mathbf{m})} \sum_{j<k} \gamma_{j k} \sum_{\nu=1}^{\infty} \nu \alpha_{\mathbf{n}}\left(\mathbf{m}-\nu \mathbf{E}_{j k}\right), \quad \alpha_{\mathbf{n}}(\mathbf{n})=1
$$

using the notation in (15), provided that $b_{\mathbf{n}}(\mathbf{m}) \neq 0$ for all $\mathbf{m} \prec \mathbf{n}$. The latter is guaranteed by our assumption in (16). We can solve this recursion relation by iteration (see Appendix $\mathrm{C}$ ). We thus obtain

$$
\alpha_{\mathbf{n}}(\mathbf{m})=\sum_{s=0}^{\infty} \alpha_{\mathbf{n}}^{(s)}(\mathbf{m})
$$

where

$$
\alpha_{\mathbf{n}}^{(0)}(\mathbf{m})=\delta_{\mathbf{n}}(\mathbf{m}), \quad \alpha_{\mathbf{n}}^{(s)}(\mathbf{m})=\frac{1}{b_{\mathbf{n}}(\mathbf{m})} \sum_{j<k} \gamma_{j k} \sum_{\nu=1}^{\infty} \nu \alpha_{\mathbf{n}}^{(s-1)}\left(\mathbf{m}-\nu \mathbf{E}_{j k}\right)
$$

for $s=1,2, \ldots$. The formula in (18) is obtained by computing

$$
\begin{aligned}
& \alpha_{\mathbf{n}}^{(s)}(\mathbf{m})=\frac{1}{b_{\mathbf{n}}(\mathbf{m})} \sum_{j_{s}<k_{s}} \gamma_{j_{s} k_{s}} \sum_{\nu_{s}=1}^{\infty} \nu_{s} \frac{1}{b_{\mathbf{n}}\left(\mathbf{m}-\nu_{s} \mathbf{E}_{j_{s} k_{s}}\right)} \sum_{j_{s-1}<k_{s-1}} \gamma_{j_{s-1} k_{s-1}} \sum_{\nu_{s-1}=1}^{\infty} \nu_{s-1} \\
& \times \frac{1}{b_{\mathbf{n}}\left(\mathbf{m}-\sum_{\ell=s-1}^{s} \nu_{\ell} \mathbf{E}_{j_{\ell} k_{\ell}}\right)} \sum_{j_{s-2}<k_{s-2}} \gamma_{j_{s-2} k_{s-2}} \sum_{\nu_{s-2}=1}^{\infty} \nu_{s-2} \frac{1}{b_{\mathbf{n}}\left(\mathbf{m}-\sum_{\ell=s-2}^{s} \nu_{\ell} \mathbf{E}_{j_{\ell} k_{\ell}}\right)} \\
& \times \cdots \frac{1}{b_{\mathbf{n}}\left(\mathbf{m}-\sum_{\ell=2}^{s} \nu_{\ell} \mathbf{E}_{j_{\ell} k_{\ell}}\right)} \sum_{j_{1}<k_{1}} \gamma_{j_{1} k_{1}} \sum_{\nu_{1}=1}^{\infty} \nu_{1} \delta_{\mathbf{n}}\left(\mathbf{m}-\sum_{r=1}^{s} \nu_{r} \mathbf{E}_{j_{r} k_{r}}\right)
\end{aligned}
$$

and replacing $\mathbf{m}$ in the arguments of the $b_{\mathbf{n}}$ by $\mathbf{n}+\sum_{r=1}^{s} \nu_{r} \mathbf{E}_{j_{r} k_{r}}$.

To see where the eigenfunctions $P_{\mathbf{n}}$ are well-defined insert (18) in (17) and obtain

$$
\check{P}_{\mathbf{n}}(\mathbf{z})=\mathbf{z}^{\mathbf{n}^{+}}+\sum_{s=1}^{\infty} \prod_{r=1}^{s}\left(\sum_{j_{r}<k_{r}} \gamma_{j_{r} k_{r}} \sum_{\nu_{r}=1}^{\infty} \nu_{r}\right) \frac{1}{\prod_{r=1}^{s} b_{\mathbf{n}}\left(\mathbf{n}+\sum_{\ell=1}^{r} \nu_{\ell} \mathbf{E}_{j_{\ell} k_{\ell}}\right)} \mathbf{z}^{\left(\mathbf{n}+\sum_{r=1}^{s} \nu_{r} \mathbf{E}_{j_{r} k_{r}}\right)^{+}}
$$

where use the notation $\check{f}_{\mathbf{m}}(\mathbf{z})=\mathbf{z}^{\mathbf{m}^{+}}$. We can estimate this using (16),

$$
\begin{aligned}
\left|\check{P}_{\mathbf{n}}(\mathbf{z})\right| & \leq\left|\mathbf{z}^{\mathbf{n}^{+}}\right|+\sum_{s=1}^{\infty} \prod_{r=1}^{s}\left(\sum_{j_{r}<k_{r}}\left|\gamma_{j_{r} k_{r}}\right| \sum_{\nu_{r}=1}^{\infty} \nu_{r}\right) \frac{1}{\Delta^{s}}\left|\mathbf{z}^{\mathbf{n}^{+}}\right| \prod_{r=1}^{s}\left|\frac{z_{j_{r}}}{z_{k_{r}}}\right|^{\nu_{r}} \\
& =\sum_{s=0}^{\infty}\left(\sum_{j<k}\left|\gamma_{j k}\right| \sum_{\nu=1}^{\infty} \nu\left|\frac{z_{j}}{z_{k}}\right|^{\nu} \frac{1}{\Delta}\right)^{s}\left|\mathbf{z}^{\mathbf{n}^{+}}\right|
\end{aligned}
$$

which implies absolute convergence of the series defining $P_{\mathbf{n}}$ provided that the condition in (19) holds true. 
Remark 3. As the proof above shows, the condition in (19) is sufficient but far from necessary. We believe that, if $b_{\mathbf{n}}(\mathbf{m}) \neq 0$ for all $\mathbf{m} \preceq \mathbf{n}$, then $P_{\mathbf{n}}(\mathbf{z})$ is well-defined in all of $\Omega_{N}$.

Remark 4. We call the eigenfunctions in Proposition 1 singular since they seem to diverge as $z_{j} \rightarrow z_{k}$, at least for positive coupling values: from a heuristic argument we expect that they behave like

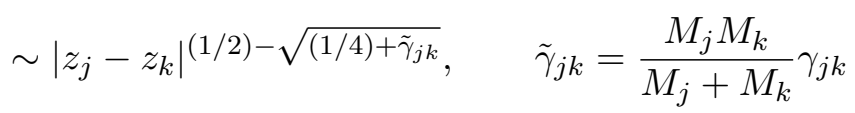

as $z_{j} \rightarrow z_{k}$. This argument suggests that these eigenfunctions are elements in $L^{2}\left([-\pi, \pi]^{N}\right)$ if all $\tilde{\gamma}_{j k}$ are negative and larger than $-1 / 4$. It would be interesting to prove that this was indeed the case since then these 'singular' eigenfunctions would be actually acceptable as quantum mechanical wave functions.

Remark 5. The idea that the (standard) CS differential operator has eigenfunctions as in (17) was probably first used by Heckman and Opdam; see e.g. [17].

Remark 6. Other remarkable special case of the generalized CS differential operator in (6) and (7) allowing for polynomial eigenfunctions are for $M_{j}=1$ for $1 \leq j \leq n<N$ and $M_{j}=-1 / \lambda$ for $n<j \leq N$, as discovered in [18] for the case $n=N-1$ and explored for general $n$ in [19,20]. In fact, all our results on the CS model eigenfunctions described here can be extended to these deformed cases [15].

The following two subsections can be skipped by readers not interested in $\mathcal{P} \mathcal{T}$-symmetric quantum mechanics.

\subsection{Exactly solvable $\mathcal{P} \mathcal{T}$-symmetric quantum many-body systems}

We now show that the eigenfunctions in Proposition 1 can be interpreted as solutions of an (partially) exactly solvable $\mathcal{P} \mathcal{T}$-symmetric quantum many-body system; see e.g. [11]. For that we observe that complex variables (3) with $\left|z_{j}\right|=R^{j}, R>0$, can be obtained by shifting the particle coordinates to the complex plane as follows,

$$
x_{j} \rightarrow x_{j}+\mathrm{i} j \epsilon
$$

and doing this replacement in the differential operator in (6) we obtain

$$
\mathcal{H}_{N}=-\sum_{j=1}^{N} \frac{1}{M_{j}} \frac{\partial^{2}}{\partial x_{j}^{2}}+\sum_{j<k} \frac{\gamma_{j k}}{4 \sin ^{2}\left(\frac{1}{2}\left(x_{j}-x_{k}-\mathrm{i}[k-j] \epsilon\right)\right)} .
$$

It is interesting to note that this defines an operator on the Hilbert space $L^{2}\left([-\pi, \pi]^{N}\right)$ which is not hermitean but rather obeys

$$
\mathcal{H}_{N}^{*}=\overline{\mathcal{H}_{N}}
$$

where the star means Hilbert space adjungation and the bar complex conjugation. However, this differential operator is invariant under the following combined parity $(\mathcal{P})$ and time reversal $(\mathcal{T})$ transformation [11]:

$$
\mathcal{P} \mathcal{T}: \quad x_{j} \rightarrow-x_{j}, \quad \mathrm{i} \rightarrow-\mathrm{i}
$$

implying $p_{j}=-\mathrm{i} \partial / \partial x_{j} \rightarrow p_{j}$. Moreover, Proposition 1 provides exact eigenfunctions of this differential operators, and the corresponding eigenvalues are indeed real, as they should be [11]: 
Corollary 1. The functions in (20) with

$$
\mathbf{z}^{\mathbf{m}^{+}}=\mathrm{e}^{\sum_{j=1}^{N}\left(\mathrm{i} x_{j}-j \epsilon\right)\left(m_{j}+s_{j}\right)}
$$

and $b_{\mathbf{n}}(\mathbf{m})$ in (15) are exact formal ${ }^{2}$ eigenfunctions of the $\mathcal{P} \mathcal{T}$-symmetric Hamiltonian $\mathcal{H}_{N}$ in (21) corresponding to the eigenvalue $E_{\mathbf{n}}$ in (14). Moreover, this eigenfunction is a welldefined function in $L^{2}\left([-\pi, \pi]^{N}\right)$ provided that (19) holds true for some $\Delta>0$ and

$$
\sum_{j<k}\left|\gamma_{j k}\right| \frac{\mathrm{e}^{-2(k-j) \epsilon}}{\left(1+\mathrm{e}^{-2(k-j) \epsilon}\right)^{2}}<\Delta .
$$

Sketch of proof. The only non-trivial part is to verify the sufficient condition for square integrability: compute

$$
\int_{[-\pi, \pi]^{N}} d^{N} x\left|\check{P}_{\mathbf{n}}(\mathbf{x})\right|^{2}=\sum_{\mathbf{m}}\left|\alpha_{\mathbf{n}}(\mathbf{m})\right|^{2} \mathrm{e}^{-2\left(m_{j}+s_{j}\right) j \epsilon},
$$

$\alpha_{\mathbf{n}}(\mathbf{m})$ in (18), and majorize by a geometric series, similarly as in the proof of Proposition 1 above.

It is interesting to note that for the CS differential operator in (1) and $s_{j}$ in (27) below, the condition in (23) holds true automatically for all partitions $\mathbf{n}$ and sufficiently large $\epsilon$ (e.g. any value $\epsilon \geq \log (R)$ with $R$ in (30) will do), as shown in the next section (see Lemma 3 and its proof in Appendix B.2).

If for such a $\mathcal{P} \mathcal{T}$-symmetric Hamiltonian the exact eigenfunction in Corollary 1 exists for all integer vectors $\mathbf{n}$, then these eigenfunctions provide a complete basis in the Hilbert space (it is not difficult to prove this, using that the functions $\check{f}_{\mathbf{n}}(\mathbf{z}), \mathbf{n} \in \mathbb{Z}^{N}$, are a complete basis). In this case we have an exactly solvable model, otherwise we only might have an partially solvable model. However, we believe that the condition in (19) can be relaxed and that it is possible to compute all eigenfunctions explicitly, i.e., the $\mathcal{P} \mathcal{T}$-symmetric Hamiltonian in (6) with eigenfunctions of the form (17) is always an exactly solvable model.

Note that we have obtained for the differential operator in (21) a family of eigenfunctions depending on parameters $s_{j}$ which we are free to choose. This is similar to what is wellknown for hermitean operators: a differential operator does not uniquely determine a self-adjoint operator, but there is usually a whole family of self-adjoint extensions labeled by continuous parameters; see e.g. [21]. It is interesting to note that one can easily construct a much larger family of $\mathcal{P} \mathcal{T}$-symmetric Hamiltonians corresponding to the differential operator in (21). In the following section we outline this construction, but our discussion is somewhat sketchy. We only include it here in the hope that it might be of interest in the context of $\mathcal{P} \mathcal{T}$-symmetric quantum mechanics [11].

\subsection{Generalized $\mathcal{P} \mathcal{T}$-symmetric quantum many-body system}

This section contains a sketch how to construct eigenfunctions the the generalized CS differential operator in (6) depending on $N(N+1) / 2$ parameters $s_{j}$ and $\lambda_{j k}, 1 \leq j<k \leq N$ (the eigenfunctions in Proposition (1) correspond to the special case $\lambda_{j k}=0$ ).

Define

$$
\Psi_{0}(\mathbf{x})=\prod_{j<k} \sin \left(\frac{1}{2}\left(x_{j}-x_{k}-\mathrm{i}[k-j] \epsilon\right)\right)^{\lambda_{j k}}
$$

\footnotetext{
${ }^{2}$ The qualifier 'formal' here means that questions of convergence are ignored.
} 
with arbitrary real parameters $\lambda_{j k}$. Then the ansatz

$$
\Psi(\mathbf{x})=\Psi_{0}(\mathbf{x}) \check{P}(\mathbf{z})
$$

in the eigenvalue equation $\mathcal{H}_{N} \Psi=E \Psi$ is equivalent to $\mathcal{H}_{N}^{\prime} \check{P}=E^{\prime} \check{P}$ with

$$
\begin{aligned}
\mathcal{H}_{N}^{\prime}= & -\sum_{j=1}^{N} \frac{1}{M_{j}} \frac{\partial^{2}}{\partial x_{j}^{2}}+\sum_{j<k}\left(\frac{\gamma_{j k}^{\prime}}{4 \sin ^{2}\left((1 / 2)\left(x_{j}-x_{k}-\mathrm{i}[k-j] \epsilon\right)\right)}\right. \\
& \left.-\lambda_{j k} \cot \left(\frac{1}{2}\left(x_{j}-x_{k}-\mathrm{i}[k-j] \epsilon\right)\right)\left(\frac{1}{M_{j}} \frac{\partial}{\partial x_{j}}-\frac{1}{M_{k}} \frac{\partial}{\partial x_{k}}\right)\right), \\
\gamma_{j k}^{\prime}= & \gamma_{j k}-\frac{M_{j}+M_{k}}{M_{j} M_{k}} \lambda_{j k}\left(\lambda_{j k}-1\right)
\end{aligned}
$$

and $E^{\prime}=E-E_{0}$ with

$$
E_{0}=\sum_{j<k<\ell} \frac{1}{2 M_{j}} \lambda_{j k} \lambda_{j \ell}-\sum_{j \neq k} \frac{1}{4 M_{j}} \lambda_{j k}^{2} .
$$

Note that for the special case where $\lambda_{j k}=\lambda M_{j} M_{k}, \gamma_{j k}$ in (7), $\epsilon=0$, the function $\Psi_{0}(\mathbf{x})$ in (24) becomes identical to the one in $(8), \gamma_{j k}^{\prime}=0$, and $E_{0}$ is identical to $\mathcal{E}_{0}$ in (9). We thus we recover (10). Another interesting case is $M_{j}=1, \lambda_{j k}=\lambda, \gamma_{j k}=2 \lambda(\lambda-1)$ in which case $\mathcal{H}_{N}^{\prime}$ becomes equal to the reduced Hamiltonian used by Sutherland in his solution of the CS model [2]. By expanding the cot- and $1 / \sin ^{2}$-terms in power series one can compute the action of $\mathcal{H}_{N}^{\prime}$ on the functions in (12) which, again, is triangular, and one then can construct eigenfunctions of the form (17), similar as in Section 3.1. One thus finds the following generalization of Proposition (1):

Proposition 2. The differential operator in (6) has formal eigenfunctions labeled by $\mathbf{n} \in \mathbb{Z}^{N}$ and corresponding to the eigenvalues

$$
E_{\mathbf{n}}=\sum_{j=1}^{N} \frac{\left(n_{j}+s_{j}\right)^{2}}{M_{j}}+\sum_{j<k} \lambda_{j k}\left(\frac{\left(n_{j}+s_{j}\right)}{M_{j}}-\frac{\left(n_{k}+s_{k}\right)}{M_{k}}\right)+E_{0}
$$

with $E_{0}$ in (25). These formal eigenfunctions are given by

$$
\Psi_{\mathbf{n}}(\mathbf{x})=\Psi_{0}(\mathbf{x}) \check{P}_{\mathbf{n}}(\mathbf{z})
$$

with $\Psi_{0}(\mathbf{x})$ in (24) and $\check{P}_{\mathbf{n}}(\mathbf{z})$ in (17) where the coefficients are determined by the following recursion relations,

$$
\left(E_{\mathbf{n}}-E_{\mathbf{m}}\right) \alpha_{\mathbf{n}}(\mathbf{m})=\sum_{j<k} \sum_{\nu=1}^{\infty}\left(\lambda_{j k}\left(\frac{\left(m_{j}+s_{j}+\nu\right)}{M_{j}}-\frac{\left(m_{k}+s_{k}-\nu\right)}{M_{k}}\right)-\nu \gamma_{j k}^{\prime}\right) \alpha_{\mathbf{n}}(\mathbf{m})
$$

with $\alpha_{\mathbf{n}}(\mathbf{n})=1$.

Note that the recursion relations in (26) still have triangular form and thus can, in principle, be solved by the method explained in Appendix $\mathrm{C}$ provided there are no degeneracies, i.e. $E_{\mathbf{n}} \neq E_{\mathbf{m}}$ for all pertinent $\mathbf{m} \preceq \mathbf{n}$. However, the resulting formula will be much more involved and probably not very illuminating.

It is interesting to note that, if

$$
\frac{n_{j}+s_{j}}{M_{j}}>\frac{n_{k}+s_{k}}{M_{k}} \quad \forall j<k,
$$

then the eigenvalues in (5) can be written in the following simple form,

$$
E_{\mathbf{n}}=\sum_{j=1}^{N} \frac{p_{j}^{2}}{M_{j}}, \quad p_{j}=n_{j}+\frac{1}{2} \sum_{k<j} \lambda_{j k}-\frac{1}{2} \sum_{k>j} \lambda_{j k}
$$




\section{A method to solve the CS model}

In our discussion of the singular eigenfunctions all generalized CS differential operators in (6) could be treated on equal footing. It thus seems natural to ask: is there anything special about the CS differential operator in (1) with regard to these singular eigenfunctions? To answer this question we compare the eigenvalues in (5) and (14) and observe that, if we choose

$$
s_{j}=\frac{1}{2}(N+1-2 j) \lambda
$$

and restrict the integer vectors $\mathbf{n}$ to partitions, then the singular eigenfunctions of $H_{N}$ in (17) not only are labeled by the same quantum numbers but also have the very same eigenvalues as its regular eigenfunctions in (2). Moreover, these singular eigenfunctions are well-defined, i.e., the condition in (16) is automatically fulfilled. We now show that this is no coincidence: The CS differential operator $H_{N}$ is special since there exists an integral operator $\hat{F}_{N}$ which commutes with it and which transforms its singular eigenfunctions in (17) as specified above into regular ones as in (2). Thus we can obtain all the regular eigenfunctions of the CS model by first constructing singular eigenfunctions (which can be done explicitly), and then transforming them using the operator $\hat{F}_{N}$. As shown below, in this way we recover the explicit formulas for Jack polynomials derived previously in [7].

In the following we make these statements more precise. For that we first define the class of functions $\mathcal{R}_{N}$ and $\mathcal{S}_{N}$ to which the regular- and singular eigenfunctions of the CS differential operator belongs.

Definition 1. The regular domain $\mathcal{R}_{N}$ of the CS differential operator in (1) is the vector space of all functions of the form $\Psi_{0}(\mathbf{x}) P(\mathbf{z})$ with $\Psi_{0}(\mathbf{x})$ in (4) and $P(\mathbf{z})$ a symmetric function in the variables $z_{j}=\mathrm{e}^{\mathrm{i} x_{j}}$ which is analytic and bounded on the domain $\left|z_{j}\right|=1 \forall j$.

Definition 2. The singular domain $\mathcal{S}_{N}$ of the CS differential operator in (1) is the vector space of all Laurent series of the form

$$
\sum_{\hat{\boldsymbol{\mu}} \in C_{-}^{N}} a_{\hat{\boldsymbol{\mu}}} \check{f}_{\mathbf{n}+\hat{\boldsymbol{\mu}}}(\mathbf{z})
$$

for some fixed $\mathbf{n} \in \mathbb{Z}^{N}$, with $\check{f}_{\mathbf{m}}$ in (12), $s_{j}$ in (27), and coefficients $a_{\hat{\boldsymbol{\mu}}}$ such that

$$
\sum_{\hat{\boldsymbol{\mu}} \in C_{-}^{N}}\left|a_{\hat{\boldsymbol{\mu}}}\right| \prod_{j<k}^{N} R^{-\mu_{j k}(k-j)}<\infty
$$

for some $R>1$ which is sufficiently large.

Remark 7. The convergence condition in (29) is chosen such that the series in (28) is absolutely convergent in the region where $\left|z_{j}\right|=R^{j} \forall j$, and it then obviously is convergent for $\left|z_{j}\right| \geq R^{j} \forall j$. We introduce this parameter $R>1$ to avoid technicalities: our crude arguments in Appendix B.2 show that the following value of $R$ is sufficiently large,

$$
R=\max (2, N(N-1)|\lambda-1| / 8),
$$

but we suspect that any value $R>1$ would do.

We now summarize the properties of the singular eigenfunctions of the CS differential operator following from Proposition 1. As mentioned, the non-trivial part is that the conditions in (19) are automatically fulfilled. 
Lemma 3. For all partitions $\mathbf{n}$ of length $N$ and $s_{j}$ as in (27) the series $\check{P}_{\mathbf{n}}$ in (17) and (18) for $\gamma_{j k}=2 \lambda(\lambda-1)$ and $M_{j}=1$ (independent of $j, k$ ) are well-defined singular eigenfunctions of the CS differential operator (1) corresponding to the eigenvalue (5):

$$
\check{P}_{\mathbf{n}} \in \mathcal{S}_{N} \text { and } H_{N} \check{P}_{\mathbf{n}}=E_{\mathbf{n}} \check{P}_{\mathbf{n}}
$$

(The proof is given in Appendix B.2.)

We now explain how to construct the operator $\hat{F}_{N}$. The idea is to use the function $F_{N}(\mathbf{x}, \mathbf{y})$ in (11) as integral kernel and define

$$
\hat{F}_{N}(\check{P})(\mathbf{x})=\int_{\mathcal{C}} \frac{d^{N} y}{(2 \pi)^{N}} F(\mathbf{x}, \mathbf{y}) \check{P}(\boldsymbol{\xi}), \quad \xi_{j}=\mathrm{e}^{\mathrm{i} y_{j}}
$$

for suitable choices of the constants $P$ and $c$, and the integration domain $\mathcal{C}$. Then Lemma 1 guarantees that this operator commutes with $H_{N}$, and it maps eigenfunctions of $H_{N}$ into eigenfunctions of $H_{N}$ without changing the eigenvalues, even though the character of these eigenfunctions is very different. A straightforward computation (deferred to Appendix B.3) then shows how $c, P$, and $\mathcal{C}$ need to be chosen to get a simple and meaningful result, and by inspection one finds the domain and range of this operator. To be precise:

Lemma 4. The prescription

$$
\hat{F}_{N}(\check{P})(\mathbf{x})=\Psi_{0}(\mathbf{x}) \prod_{j=1}^{N}\left(\oint_{\left|\xi_{j}\right|=R^{j}} \frac{d \xi_{j}}{2 \pi \mathrm{i} \xi_{j}}\right) \frac{\prod_{1 \leq j<k \leq N}\left(1-\xi_{j} / \xi_{k}\right)^{\lambda}}{\prod_{j, k=1}^{N}\left(1-z_{j} / \xi_{k}\right)^{\lambda}} \prod_{j=1}^{N} \xi_{j}^{-s_{j}} \check{P}(\boldsymbol{\xi})
$$

with $s_{j}$ in (27) and $R>1$ sufficiently large, defines an operator commuting with the CS differential operator (1) and mapping its singular- to its regular domain:

$$
\hat{F}_{N}\left(H_{N} \check{P}\right)(\mathbf{x})=H_{N} \hat{F}_{N}(\check{P})(\mathbf{x})
$$

and

$$
\hat{F}_{N}(\check{P}) \in \mathcal{R}_{N} \text { for all } \check{P} \in \mathcal{S}_{N} \text {. }
$$

(The proof is given in Appendix B.3.)

Combining Lemmas 3 and 4 above we can conclude that the functions $\hat{F}_{N}\left(\hat{P}_{\mathbf{n}}\right)(\mathbf{x})$ are regular eigenfunctions of the CS differential operator $H_{N}$ to the eigenvalues in (5), and they are of the form

$$
\hat{F}_{N}\left(\hat{P}_{\mathbf{n}}\right)(\mathbf{x})=\Psi_{0}(\mathbf{x}) P_{\mathbf{n}}(\mathbf{z}) .
$$

One can prove that $P_{\mathbf{n}}(\mathbf{z})$ is actually a symmetric polynomial of degree $\sum_{j=1}^{N} n_{j}$ and that $P_{\mathbf{n}}(\mathbf{z})$ is equal to the corresponding Jack polynomial, up to normalization; see [5] and [15] for details. Interchanging summation and the integral transform we thus obtain the following representation of the Jack polynomials as a linear superposition of the special symmetric polynomials $f_{\mathbf{n}}=$ $\hat{F}_{N}\left(\check{f}_{\mathbf{n}}\right)$ : 
Proposition 3. The Jack polynomial labeled by the partition $\mathbf{n}$ is proportional to

$$
P_{\mathbf{n}}(\mathbf{z})=\sum_{\mathbf{m} \preceq \mathbf{n}} \alpha_{\mathbf{n}}(\mathbf{m}) f_{\mathbf{n}}(\mathbf{z})
$$

where

$$
\begin{aligned}
f_{\mathbf{m}}(\mathbf{z}) & =\prod_{j=1}^{N}\left(\oint_{\left|\xi_{j}\right|=R^{j}} \frac{d \xi_{j}}{2 \pi \mathrm{i} \xi_{j}} \xi_{j}^{m_{j}}\right) \frac{\prod_{1 \leq j<k \leq N}\left(1-\xi_{j} / \xi_{k}\right)^{\lambda}}{\prod_{j, k=1}^{N}\left(1-z_{j} / \xi_{k}\right)^{\lambda}}, \\
\alpha_{\mathbf{n}}(\mathbf{m}) & =\delta_{\mathbf{n}}(\mathbf{m})+\sum_{s=1}^{\infty}[2 \lambda(\lambda-1)]^{s} \prod_{r=1}^{s}\left(\sum_{j_{r}<k_{r}} \sum_{\nu_{r}=1}^{\infty} \nu_{r}\right) \frac{\delta_{\mathbf{n}}\left(\mathbf{m}-\sum_{r=1}^{s} \nu_{r} \mathbf{E}_{j_{r} k_{r}}\right)}{\prod_{r=1}^{s} b_{\mathbf{n}}\left(\mathbf{n}+\sum_{\ell=1}^{r} \nu_{\ell} \mathbf{E}_{j_{\ell} k_{\ell}}\right)}
\end{aligned}
$$

and $b_{\mathbf{n}}(\mathbf{m})=E_{\mathbf{n}}-E_{\mathbf{m}}$ with $E_{\mathbf{n}}$ in $(5)$.

Remark 8. A non-trivial point in the result above is possible degeneracies: if the partition $\mathbf{n}$ is such that there is no other partition $\mathbf{m}$ such that $\sum_{j} m_{j}=\sum_{j} n_{j}$ and $E_{\mathbf{n}}=E_{\mathbf{m}}$ then the corresponding eigenfunction of the CS model is (essentially) unique and the symmetric polynomials thus only can be equal to the corresponding Jack polynomial. However, if this is not the case an additional argument is needed.

Remark 9. It is interesting to note that, in the special case $\lambda=1$, the result above gives the following integral representation of the Schur polynomials,

$$
S_{\mathbf{n}}(\mathbf{z})=\prod_{j=1}^{N}\left(\oint_{\left|\xi_{j}\right|=R^{j}} \frac{d \xi_{j}}{2 \pi \mathrm{i} \xi_{j}} \xi_{j}^{n_{j}}\right) \frac{\prod_{1 \leq j<k \leq N}\left(1-\xi_{j} / \xi_{k}\right)}{\prod_{j, k=1}^{N}\left(1-z_{j} / \xi_{k}\right)} ;
$$

this is a simple consequence of results in [22] (see [15]); see also Appendix B in [23].

Remark 10. Integral representations of the Jack polynomials involving integral operators somewhat similar to ours were previously obtained in [24, 25].

\section{Epilogue}

Yuri Suris at a meeting in Rome in May 2001 pointed out to me that the (elliptic generalization) of the identity in Lemma 1 looks very much like the Bäcklund transformation for the classical Calogero-Sutherland system, but I did not absorb this comment at that time. In January 2006 in Rome again it eventually 'sank in' and I eventually learned about Stefan Rauch's pioneering work on this [26]. I thus understand now that it was not a coincidence that it was through Stefan that I got to know Vadim who has played a central role in developing the idea of quantum Bäcklund transformations (see e.g. [9]) which, in the special case of the CS model, is based on the very same identity. I stumbled over this identity by coincidence when working on a project where I studied a quantum field theory model (initially with Alan Carey) with the aim to learn more about the so-called fractional quantum Hall effect [4], and through the positive feedback and encouragement I received from the integrable system's community I started to explore its consequences, and now I find myself working more and more on problems related to the mathematical theory of special functions. This happened to a large degree due to Vadim. He has encouraged me and given me a few glimpses on some of his visions for which I am very grateful and which will probably continue to have a strong influence on my work. There will be probably more papers in the future where Vadim's name should be mentioned ... 


\section{A Groundstate of the generalized CS model}

As discussed in the text, the Schrödinger operator in (6) is a partially solvable system in the sense that its exact groundstate and corresponding groundstate energy can be found explicitly. In this appendix we give a precise formulation and proof of this fact.

Lemma 5. Let $\Phi_{0}(\mathbf{x})$ be as in (8) and

$$
Q_{j}^{ \pm}= \pm \frac{\partial}{\partial x_{j}}+\mathcal{V}_{j} \quad \text { with } \quad \mathcal{V}_{j}=\frac{\partial \log \Phi_{0}(\mathbf{x})}{\partial x_{j}}
$$

for arbitrary complex parameters $\lambda$ and $M_{j}$. Then

$$
\sum_{j=1}^{N} \frac{1}{M_{j}} Q_{j}^{+} Q_{j}^{-}=\mathcal{H}_{N}-\mathcal{E}_{0}
$$

with $\mathcal{H}_{N}$ the differential operator in (6) and $\mathcal{E}_{0}$ the constant in (9), and this implies (10). In particular, if all $M_{j}$ are positive then $\mathcal{H}_{N}$ defines a self-adjoint operator on the Hilbert space $L^{2}\left([-\pi, \pi]^{N}\right)$ bounded from below by $\mathcal{E}_{0}$ (Friedrichs extension; see e.g. [21]), and $\Phi_{0}$ is its groundstate.

Proof. We compute

$$
\mathcal{V}_{j}=\sum_{k \neq j} M_{j} M_{k} \lambda \phi\left(x_{j}-x_{k}\right) \text { with } \phi(z):=\frac{1}{2} \cot \left(\frac{z}{2}\right)
$$

and thus, using

$$
\phi^{\prime}(z)=-\frac{1}{4}-\phi(z)^{2}=-\frac{1}{4} \sin ^{-2}\left(\frac{z}{2}\right)
$$

we obtain by straightforward computations

$$
\sum_{j=1}^{N} \frac{1}{M_{j}} Q_{j}^{+} Q_{j}^{-}=\sum_{j=1}^{N} \frac{1}{M_{j}}\left(-\frac{\partial^{2}}{\partial x_{j}^{2}}+\mathcal{V}_{j}^{2}+\frac{\partial \mathcal{V}_{j}}{\partial x_{j}}\right)=\mathcal{H}_{N}-(*)
$$

with $\mathcal{H}_{N}$ in (6) and the reminder terms

$$
(*)=\lambda^{2}\left(-\sum_{j} \sum_{k \neq j} \sum_{\ell \neq j, k} M_{j} M_{k} M_{\ell} \phi\left(x_{j}-x_{k}\right) \phi\left(x_{j}-x_{\ell}\right)+\frac{1}{4} \sum_{j} \sum_{k \neq j} M_{j} M_{k}^{2}\right) .
$$

The non-trivial part of the result thus is that the three-body terms in $(*)$ add up to a constant. To see this we symmetrize the first sum in $(*)$ above as follows,

$$
-2 \sum_{j<k<\ell} M_{j} M_{k} M_{\ell}\left[\phi\left(x_{j}-x_{k}\right) \phi\left(x_{j}-x_{\ell}\right)+\phi\left(x_{k}-x_{j}\right) \phi\left(x_{k}-x_{\ell}\right)+\phi\left(x_{\ell}-x_{j}\right) \phi\left(x_{\ell}-x_{k}\right)\right] .
$$

This allows us to use the following trigonometric identity

$$
\phi\left(x_{j}-x_{k}\right) \phi\left(x_{j}-x_{\ell}\right)+\phi\left(x_{k}-x_{j}\right) \phi\left(x_{k}-x_{\ell}\right)+\phi\left(x_{\ell}-x_{j}\right) \phi\left(x_{\ell}-x_{k}\right)=-\frac{1}{4}
$$

and obtain

$$
(*)=\frac{\lambda^{2}}{4}\left(2 \sum_{j<k<\ell} M_{j} M_{k} M_{\ell}+\sum_{j<k} M_{j} M_{k}\left(M_{j}+M_{k}\right)\right),
$$

and by straightforward computations we find $(*)=\mathcal{E}_{0}$ as in $(9)$.

Since obviously $Q_{j}^{-} \Phi_{0}=0$ for all $j$ this proves (10). Moreover, $Q_{j}^{+}$is the hermitean conjugate of $Q_{j}^{-}$, and if all $M_{j}>0$ then $\sum_{j}\left(1 / M_{j}\right) Q_{j}^{+} Q_{j}^{-}$therefore defines obviously a non-negative, selfadjoint operator. This implies $\mathcal{H}_{N} \geq \mathcal{E}_{0}$, and thus $\Phi_{0}$ is groundstate of $\mathcal{H}_{N}$. 


\section{B Proofs}

\section{B.1 Proof of Lemma 1}

The identity in (10) holds obviously true for arbitrary real parameters $M_{j}$ and complex variables $x_{j}$ (see Appendix A).

We double the degrees of freedom and replace $N$ by $2 N$. Setting $M_{j}=1, M_{N+j}=-1$, and $x_{N+j}=y_{j}$ for $j=1,2, \ldots, N$ we find that $\mathcal{H}_{2 N}=H_{N}(\mathbf{x})-H_{N}(\mathbf{y})$ (note that all interaction terms mixing the variables $x_{j}$ and $y_{j}$ are zero since $\left(M_{j}+M_{N+k}\right)=0$ for $j, k=1,2, \ldots, N$; we also slightly abuse notation here), $\Phi_{0}(\mathbf{x}, \mathbf{y})=F_{N}(\mathbf{x}, \mathbf{y})$ for $P=0$, and $\mathcal{E}_{0}=0$. Thus the identity in (10) implies (11) for $P=0$ and $c=1$.

We now observe that, due to translation invariance, $H_{N}(\mathbf{x})$ has the following simple dependence on the center of mass coordinate $X=\sum_{j=1}^{N} x_{j} / N$,

$$
H_{N}(\mathbf{x})=-\frac{\partial^{2}}{\partial X^{2}}+\cdots
$$

where the dots are terms independent of $X$ (they only depend on the relative coordinates $x_{j}-x_{N}$ for $\left.N=1,2, \ldots, N-1\right)$. This implies that the identity in (11) is invariant under the transformation

$$
F_{N}(\mathbf{x}, \mathbf{y}) \rightarrow c \mathrm{e}^{\mathrm{i} P^{\prime}(X-Y)} F_{N}(\mathbf{x}, \mathbf{y})
$$

for arbitrary constants $P^{\prime}=P N$ and $c$ (the invariance under multiplication with $c$ is trivial, of course).

\section{B.2 Proof of Lemma 3}

We observe that

$$
b_{\mathbf{n}}\left(\mathbf{n}+\sum_{j<k} \mu_{j k} \mathbf{E}_{j k}\right)=\sum_{j=1}^{N}\left(\sum_{k=j+1}^{N} \mu_{j k}\left[n_{j}-n_{k}+(k-j) \lambda\right]+\left[\sum_{k<j} \mu_{k j}-\sum_{k>j} \mu_{j k}\right]^{2}\right)
$$

which shows that (16) holds true with $\Delta=2 \lambda$. By a simple computation we find that (19) is equivalent to

$$
\sum_{j<k} \frac{\left|z_{j} / z_{k}\right|}{\left(1-\left|z_{j} / z_{k}\right|\right)^{2}}<\frac{1}{|\lambda-1|}
$$

Replacing $\left|z_{j}\right|$ by $R^{j}$ we find that (29) is equivalent to

$$
\sum_{j<k} \frac{R^{j-k}}{\left(1-R^{j-k}\right)^{2}}<\frac{1}{|\lambda-1|}
$$

and this holds true for sufficiently large values of $R>1$, e.g. by the following rough estimates

$$
\text { 1.h.s. of }(33) \leq \sum_{j<k} \frac{R^{-1}}{4}=\frac{R^{-1} N(N-1)}{8} \text {, }
$$

assuming $R \geq 2$, we find that the $R$ given in (30) will do. 


\section{B.3 Proof of Lemma 4}

We express the function $\left.F_{(} \mathbf{x}, \mathbf{y}\right)$ in terms of the variables $z_{j}=\mathrm{e}^{\mathrm{i} x_{j}}$ and $\xi_{j}=\mathrm{e}^{\mathrm{i} y_{j}}($ using $\sin (x / 2)=$ $\left.z^{1 / 2}(1-1 / z) /(2 \mathrm{i})\right)$, and by a straightforward computation we find

$$
\begin{aligned}
F_{N}(\mathbf{x}, \mathbf{y})= & c \Psi_{0}(\mathbf{x})(2 \mathrm{i})^{\lambda\left(N^{2}-N(N-1) / 2\right)}\left(\prod_{j=1}^{N}\left(z_{j}^{P+N \lambda / 2} \xi_{j}^{-P-(N+1-2 j) \lambda / 2-N \lambda / 2}\right)\right) \\
& \times \frac{\prod_{1 \leq j<k \leq N}\left(1-\xi_{j} / \xi_{k}\right)^{\lambda}}{\prod_{j, k=1}^{N}\left(1-z_{j} / \xi_{k}\right)^{\lambda}} \prod_{j=1}^{N} \xi_{j}^{-s_{j}} .
\end{aligned}
$$

To get rid of an awkward constant and the non-analytical (for non-integer $\lambda$ ) factors $z_{j}$ we choose

$$
c=(2 \mathrm{i})^{\lambda\left(N(N-1) / 2-N^{2}\right)} \quad \text { and } \quad P=-\frac{\lambda N}{2} .
$$

This yields

$$
F_{N}(\mathbf{x}, \mathbf{y})=\Psi_{0}(\mathbf{x}) \frac{\prod_{1 \leq j<k \leq N}\left(1-\xi_{j} / \xi_{k}\right)^{\lambda}}{\prod_{j, k=1}^{N}\left(1-z_{j} / \xi_{k}\right)^{\lambda}} \prod_{j=1}^{N} \xi_{j}^{-\lambda(N+1-2 j) / 2} .
$$

From this we see that if we choose the integration paths as

$$
\mathcal{C}: \quad y_{j}=\phi_{j}-\mathrm{i} j \epsilon, \quad-\pi \leq \phi_{j} \leq \pi \quad \text { for } \quad j=1,2, \ldots, N
$$

then $\xi_{j}=R^{j} \mathrm{e}^{\mathrm{i} \phi_{j}}$ with $R=\mathrm{e}^{\epsilon}>0$, and thus we find that (31) is identical with what is given in $(32)$.

It is important to note that $\hat{F}_{N}\left(\check{f}_{\mathbf{n}}\right)$ for monomials in (12) is well-defined if and only if $s_{j}$ is chosen as in (27) (modulo integers, of course), and $\mathcal{S}_{N}$ is the natural domain for $\hat{F}_{N}$ since it contains all linear superpositions of these $\check{f}_{\mathbf{n}}(\mathbf{z})$ which are absolutely convergent on $\mathcal{C}$ (recall (28), $\left|\mathbf{z}^{\mathbf{m}}\right|=\prod_{j} R^{j m_{j}}$ on $\mathcal{C}$, and (29)): writing $\hat{F}_{N}(\check{P})(\mathbf{x})=\Psi_{0}(\mathbf{x}) P(\mathbf{z})$,

$$
P(\mathbf{z})=\prod_{j=1}^{N}\left(\oint_{\left|\xi_{j}\right|=R^{j}} \frac{d \xi_{j}}{2 \pi \mathrm{i} \xi_{j}}\right) \frac{\prod_{1 \leq j<k \leq N}\left(1-\xi_{j} / \xi_{k}\right)^{\lambda}}{\prod_{j, k=1}^{N}\left(1-z_{j} / \xi_{k}\right)^{\lambda}} \prod_{j=1}^{N} \xi_{j}^{-s_{j}} \check{P}(\boldsymbol{\xi})
$$

we can estimate on $\left|z_{j}\right|=1$,

$$
|P(\mathbf{z})| \leq \frac{\prod_{1 \leq j<k \leq N}\left(1-R^{j-k}\right)^{\lambda}}{\prod_{k=1}^{N}\left(1-R^{-k}\right)^{N \lambda}} \prod_{j=1}^{N} R^{-j s_{j}}|\check{P}(\boldsymbol{\xi})|
$$

and thus prove that $\hat{F}_{N}(\check{P})(\mathbf{x})$ is in the regular domain $\mathcal{R}_{N}$ provided $\check{P}$ is in the singular domain $\mathcal{S}_{N}$. 


\section{Explicit diagonalization of triangular matrices}

In this Appendix we explain the method to explicitly diagonalize a non-degenerate triangular matrix which we use in the main text. The matrix in the main text is indexed by integer vectors, but to simplify our notation here we label the matrix elements by integers $J, K \in \mathbb{Z}$, i.e., we write the matrix as $A=\left(A_{J K}\right)_{J, K \in \mathbb{Z}}$.

We are interested in finding the eigenvector $v=\left(v_{J}\right)_{J \in \mathbb{Z}}$ of this matrix $A$ corresponding to the eigenvalue $E=A_{L L}$. Our assumptions about the matrix are as follows,

$$
A_{J K} \neq 0 \quad \text { only if } \quad K \succeq J, \quad A_{J J}-A_{L L} \neq 0 \quad \forall J \succ L .
$$

The symbol ' $\succeq$ ' here can mean any partial ordering of the integers. We write $a_{K}=A_{K K}$ for the eigenvalues of this matrix.

The $v_{J}$ are determined by the eigenvalue equation

$$
\sum_{K \succeq J} A_{J K} v_{K}=E v_{J}
$$

for $J \preceq L$, where $v_{K}=0$ for $K \succ L$ and $v_{L}=1$. For $J=L$ we get $E=A_{L L}=a_{L}$, and for $J \prec L$

$$
v_{J}=\frac{1}{\left(a_{L}-a_{J}\right)} \sum_{K \succ J} A_{J K} v_{K}
$$

which gives well-defined recursion relations due to our non-degeneracy assumption. We can solve this by iteration:

$$
v_{J}=\sum_{s=0}^{\infty} v_{J}^{(s)}
$$

where

$$
v_{J}^{(0)}=\delta_{J L}, \quad v_{J}^{(s)}=\frac{1}{\left(a_{L}-a_{J}\right)} \sum_{K \succ J} A_{J K} v_{K}^{(s-1)} \quad \forall s>1
$$

which is well-defined since $v_{K}^{(s-1)}=0$ for $K \succ L$. This yields

$$
\begin{aligned}
v_{J}= & \delta_{J L}+\sum_{s=1}^{\infty} \frac{1}{\left(a_{L}-a_{J}\right)} \sum_{K_{1} \succ J} A_{J K_{1}} \frac{1}{\left(a_{L}-a_{K_{1}}\right)} \sum_{K_{2} \succ K_{1}} A_{K_{1} K_{2}} \cdots \\
& \times \frac{1}{\left(a_{L}-a_{K_{s-1}}\right)} \sum_{K_{s} \succ K_{s-1}} A_{K_{s-1} K_{s}} \delta_{K_{s}, L} .
\end{aligned}
$$

We thus get the following fully explicit formula for the components of the eigenvector,

$$
v_{J}=\delta_{J L}+\sum_{s=1}^{\infty} \sum_{K_{s} \succ K_{s-1} \succ \cdots \succ K_{1} \succ J} \frac{A_{J K_{1}} A_{K_{1} K_{s}} \cdots A_{K_{s-1} K_{s}} \delta_{K_{s} L}}{\left(a_{L}-a_{K_{1}}\right)\left(a_{L}-a_{K_{2}}\right) \cdots\left(a_{L}-a_{K_{s}}\right)} .
$$

Note that we keep the sum infinite only for simplicity of notation, but it actually has only a finite number of non-zero terms: convergence is no problem here, of course.

\section{Acknowledgements}

I would like to thank Orlando Ragnisco for organizing and inviting me to several inspiring meetings in Rome and for showing me Ref. [26]. I thank Martin Hallnäs for useful comments on the manuscript and Alexander Veselov for reading the paper and several helpful comments. This work was supported by the Swedish Science Research Council (VR) and the European Union through the FP6 Marie Curie RTN ENIGMA (Contract number MRTN-CT-2004-5652). 


\section{References}

[1] Calogero F., Solution of the one-dimensional $N$-body problems with quadratic and/or inversely quadratic pair potentials, J. Math. Phys. 12 (1971), 419-436.

[2] Sutherland B., Exact results for a quantum many-body problem in one dimension. II, Phys. Rev. A5 (1972), $1372-1376$.

[3] Kuznetsov V.B., Mangazeev V.V., Sklyanin E.K., Q-operator and factorised separation chain for Jack polynomials, Indag. Math. (N.S.) 14 (2003), 451-482, math.CA/0306242.

[4] Langmann E., Anyons and the elliptic Calogero-Sutherland model, Lett. Math. Phys. 54 (2000), 279-289, math-ph/0007036.

[5] Langmann E., Algorithms to solve the (quantum) Sutherland model, J. Math. Phys. 42 (2001), 4148-4157, math-ph/0104039.

[6] Langmann E., An explicit solution of the (quantum) elliptic Calogero-Sutherland model, math-ph/0407050.

[7] Langmann E., A method to derive explicit formulas for an elliptic generalization of the Jack polynomials, in Proceedings of Workshop "Jack, Hall-Littlewood and Macdonald polynomials" (September 23-26, 2003, Edinburgh), Editors V.B. Kuznetsov and S. Sahi, Contemp. Math. 417 (2006), 257-270, math-ph/0511015.

[8] Langmann E., An algorithm to solve the elliptic Calogero-Sutherland model, math-ph/0401029.

[9] Kuznetsov V.B., Sklyanin E.K., On Bäcklund transformations for many-body systems, J. Phys. A: Math. Gen. 31, 1998, 2241-2251, solv-int/9711010.

[10] Kuznetsov V.B., Petrera M., Ragnisco O., Separation of variables and Bäcklund transformations for the symmetric Lagrange top, J. Phys. A: Math. Gen. 37 (2004), 8495-8512, nlin.SI/0403028.

[11] Bender C.M., Boettcher S., Meisinger P., PT-symmetric quantum mechanics, J. Math. Phys. 40 (1999), 2201-2229, quant-ph/9809072.

[12] Stanley R.P., Some combinatorial properties of Jack symmetric functions, Adv. Math. 77 (1989), $76-115$.

[13] Dunkl C.F., Xu Y., Orthogonal polynomials of several variables, Cambridge University Press, Cambridge, 2001.

[14] Hallnäs M., Langmann E., Explicit formulas for the eigenfunctions of the $N$-body Calogero model, J. Phys. A: Math. Gen. 39 (2006), 3511-3533, math-ph/0511040.

[15] Hallnäs M., Langmann E., Quantum Calogero-Sutherland type models associtated with orthogonal polynomials, work in progress.

[16] Sen D., A multispecies Calogero-Sutherland model, Nuclear Phys. B 479 (1996), 554-574, cond-mat/9512014.

[17] Heckman G.J., Opdam E.M., Root systems and hypergeometric functions. I, Compos. Math. 64 (1987), 329-352.

[18] Chalykh O., Feigin M., Veselov A., New integrable generalizations of Calogero-Moser quantum problem, J. Math. Phys. 39 (1998), 695-703.

[19] Sergeev A.N., Veselov A.P., Deformed quantum Calogero-Moser problems and Lie superalgebras, Comm. Math. Phys. 245 (2005), 249-278, math-ph/0303025.

[20] Sergeev A.N., Veselov A.P., Generalised discriminants, deformed Calogero-Moser-Sutherland operators and super-Jack polynomials, Adv. Math. 192 (2005), 341-375, math-ph/0307036.

[21] Reed M., Simon B., Methods of modern mathematical physics, II: Fourier analysis, self-adjointness, Academic Press, New York, 1975.

[22] Macdonald I.G., Symmetric functions and Hall polynomials, Oxford Mathematical Monographs, Clarendon Press, Oxford, 1979.

[23] Awata H., Fukuma M. Matsuo Y., Odake S., Representation theory of the $W_{1+\infty}$ algebra, Progr. Theoret. Phys. Suppl. 118 (1995), 343-374, hep-th/9408158.

[24] Mimachi K., Yamada Y., Singular vectors of the Virasoro algebra in terms of Jack symmetric polynomials, Comm. Math. Phys. 174 (1995), 447-455.

[25] Awata H., Matsuo Y., Odake S., Shiraishi J., Excited states of Calogero-Sutherland model and singular vectors of the $W_{N}$ algebra, Nuclear Phys. B 449 (1995), 347-374, hep-th/9503043.

[26] Wojciechowski S., The analogue of the Bäcklund transformation for integrable many-body systems, J. Phys. A: Math. Gen. 15 (1982), L653-L657. 